\title{
Satisfacción laboral y síndrome de burnout en docentes durante el confinamiento por la pandemia COVID-19
}

\section{Job satisfaction and burnout syndrome in teachers during confinement due to the COVID-19 pandemic}

\author{
Daisy Mily Cortez-Silva* \\ Universidad Peruana Unión, Tarapoto, Perú \\ ORCID: https://orcid.org/0000-0002-9186-2604 \\ Nila Campana Mendoza \\ Universidad Peruana Unión, Tarapoto, Perú \\ ORCID: https://orcid.org/0000-0002-2425-8124 \\ Noely Huayama Tocto \\ Universidad Peruana Unión, Tarapoto, Perú \\ ORCID: https://orcid.org/0000-0003-1245-0351 \\ Jessica Aranda Turpo \\ Universidad Peruana Unión, Tarapoto, Perú \\ ORCID: https://orcid.org/0000-0001-9976-1560
}

*Correspondencia:

Email: daisy.cortez@upeu.edu.pe
Citar como:

Cortez-Silva, D., Campana, N., Huayana, N., \& Aranda, J. (2021). Satisfacción laboral y síndrome de burnout en docentes durante el confinamiento por la pandemia COVID19. Propósitos y Representaciones, 9(3), e812. http://dx.doi.org/10.20511/pyr2021.v9n3.812 


\section{Resumen}

La sobrecarga laboral que presentan los docentes en el contexto actual es una situación de riesgo, ya que puede generar estados prolongados de estrés provocando un síndrome de burnout y afectando su satisfacción laboral. El objetivo de la investigación fue determinar la relación significativa entre la satisfacción laboral y los componentes del síndrome de burnout durante el encierro por la pandemia de COVID-19. El estudio tuvo un diseño correlacional transversal. Participaron 205 docentes, quienes respondieron la Escala de Satisfacción Laboral para Docentes (ESLA-Educación) y el Inventario de Burnout Maslach (MBI). Los resultados indican una correlación inversa estadísticamente significativa entre la satisfacción laboral y los componentes del síndrome de burnout: agotamiento emocional ( $r h o=-.315 ; \mathrm{p}=.000$ ), despersonalización (rho $=-.252 ; \mathrm{p}=.000)$ y realización personal (rho $=-.254 ; \mathrm{p}=.000)$. Se concluye que los docentes que presentan elevado cansancio emocional, pérdida de sensibilidad y empatía, deterioro de sus capacidades y sentimiento de fracaso muestran a su vez insatisfacción laboral. Por lo tanto, es necesario contar con factores de protección para prevenir la angustia emocional de los profesores.

Palabras clave: Satisfacción en el trabajo; Estrés ocupacional; Trabajo docente.

\section{Summary}

The work overload that teachers present in the current context is a risky situation, since it can generate prolonged states of stress causing a burnout syndrome and affecting their job satisfaction. The objective was to determine the significant relationship between job satisfaction and the components of burnout syndrome during confinement due to the COVID-19 pandemic. The study had a cross-sectional correlational design. 205 teachers participated, who answered the Job Satisfaction Scale for Teachers (ESLA-Education) and the Maslach Burnout Inventory (MBI). The results indicate a statistically significant inverse correlation between job satisfaction and the components of burnout syndrome: emotional exhaustion ( $\mathrm{rho}=-.315 ; \mathrm{p}=.000$ ), depersonalization (rho $=-.252 ; \mathrm{p}=.000$ ) and achievement personal (rho $=-.254 ; \mathrm{p}=.000$ ). It is concluded that teachers who present high emotional fatigue, loss of sensitivity and empathy, deterioration of their abilities and a feeling of failure show job dissatisfaction. Therefore, it is necessary to have protective factors to prevent emotional distress for teachers.

Keywords: Job satisfaction; Occupational stress; Teaching job.

\section{Introducción}

La pandemia mundial por la COVID-19 ha trastocado la educación, pues los estudiantes y los docentes se están enfrentando a distintos cambios y retos tanto a nivel personal como académicolaboral, debido a que el Ministerio de Educación a dispuesto realizar un servicio educativo no presencial o remoto (MINEDU, 2020), tal disposición ha obligado a los docentes a adaptarse a nuevas plataformas, modificar sus métodos de enseñanza y retroalimentación, además de la necesidad de contar con mayor capacitación y apoyo (Fondo de las Naciones Unidas para la Infancia, 2020) generando en los docentes sobrecarga e insatisfacción de su trabajo pues algunos no se sienten preparados para realizarlo.

Mejia et al. (2019) consideran que el estrés laboral es uno de los problemas más frecuentes que traen consecuencias desfavorables en el ambiente de trabajo. En un estudio realizado por Robalino y Körner (2005) encontraron que el 36,6\% de docentes peruanos padecen estrés laboral y un alarmante $12,7 \%$ tienen altos niveles de desgaste emocional, uno de los componentes del síndrome de burnout. Así mismo, Fernández (2002) menciona que, en 10 estudios realizados en Lima, el 43,2\% de los profesores experimentan altos niveles de burnout. Respecto a esto, 
Smetackova et al. (2019) afirman que es relevante realizar más estudios en este campo pues tiene una gran relevancia a fin de mejorar el bienestar de los docentes.

El síndrome de burnout es una respuesta duradera frente a estresores emocionales e interpersonales crónicos en el trabajo, Maslasch plantea el modelo trifactorial, caracterizado por tres manifestaciones principales, agotamiento emocional, despersonalización y baja realización personal (Maslach y Jackson, 1981). Siendo el agotamiento una de las características centrales del burnout, aquí la persona siente fastidio, agobio y cansancio por las funciones del trabajo, provocando una disminución de interés y satisfacción laboral, como también sentimientos de fracaso, abatimiento, irritabilidad y ansiedad por haber intentado modificar la situación sin un resultado satisfactorio (Jiménez et al., 2012; García et al., 2017). La despersonalización es comprendida como la falta de interés, pérdida de sensibilidad y empatía hacia los receptores del servicio y está acompañado de un incremento de irritabilidad y de una desmotivación hacia su trabajo. En cuanto a la falta de realización personal es la percepción de una disminución de logro en el trabajo juntamente con sensaciones de fracaso, baja autoestima e insatisfacción con su propia persona y con los resultados laborales (Jiménez et al., 2012).

Desde la teoría organizacional, el síndrome de burnout es una respuesta a los estresores del contexto de la organización y la falta de estrategias para afrontarlo (Gil-Monte y Peiró, 1999). Siendo relevante la salud de la organización (Cox, Kuk y Leiter, 1993) y el apoyo, cultura y clima organizacional (Winnubst, 1993). Por lo tanto, la satisfacción laboral juega un rol importante en el desarrollo y mantenimiento del burnout, Gómez-Perdomo, Meneses-Higuita y Palacio-Monte (2017) y Tomé y Ceinos (2017) coinciden en afirmar que a menor satisfacción en el contexto laboral mayores índices de burnout.

La satisfacción laboral según Cavalcante (2004) puede conceptualizarse desde dos perspectivas como un estado emocional o como una actitud generalizada con relación a su trabajo. El presente trabajo se enfoca en la segunda perspectiva, la que define a la satisfacción laboral como la actitud que el sujeto tiene hacia su trabajo. Esta actitud generalizada está compuesta por tres componentes los cuales son; cognitivo, afectivo y actitudinal. El cognitivo está relacionado con la creencia, los pensamientos y las opiniones que tiene el trabajador hacía su labor (Menghi y Oros, 2014). El afectivo tiene que ver con las emociones y los sentimientos positivos o negativos que producen los diferentes rasgos de su trabajo. Y el actitudinal es la acción que el sujeto realiza con relación a los diferentes aspectos de su trabajo. Es decir, al evaluar cómo es la vida laboral, se generarán emociones positivas o negativas y en función a eso se representará una conducta $u$ otra en el lugar de trabajo (Cantón y Téllez, 2016; Arias et al., 2019).

Desde esta perspectiva, la satisfacción laboral del docente es indispensable para lograr un trabajo productivo y saludable (Díaz y Barra, 2017) ya que los docentes se enfrentan a distintos cambios y retos a nivel institucional, llegando a convertirse en el eje central tanto para la educación como la formación de nuevas generaciones (Duche, Gutiérrez, y Paredes 2019).

Por lo cual, se pretende determinar la relación significativa entre satisfacción laboral y los componentes de síndrome de burnout en docentes de instituciones educativas de la ciudad de Moyobamba durante el confinamiento por la pandemia COVID-19.

\section{Método}

\section{Participantes}

Se realizó un muestreo no probabilístico intencional y como criterios de inclusión se consideró a docentes entre las edades de 23 a 65 años, que laboren a tiempo completo en instituciones educativas nacionales de la ciudad de Moyobamba en San Martín, Perú. Se excluyó a docentes que se negaron a participar en el estudio. 
Cumpliendo con los criterios de inclusión y exclusión participaron voluntariamente 205 docentes (60\% de mujeres y $40 \%$ de varones) que laboraban en instituciones educativas nacionales de la ciudad de Moyobamba, en San Martín, Perú. La edad promedio de los participantes fue de 42.49 años $(\mathrm{M}=2.69, \sigma=9.38)$, de los cuales un $51.7 \%$ es personal nombrado y $48.3 \%$ contratado. El $42 \%$ de los docentes enseña al nivel primario, el $40 \%$ al nivel secundario y un $18 \%$ al nivel inicial. Finalmente, el 36.6\% son titulados (véase Tabla 1).

Tabla 1.

Características sociodemográficas de los participantes

\begin{tabular}{llcc}
\hline Variable & Categoría & $\mathrm{f}$ & $\%$ \\
\hline Edad & $23-30$ & 22 & $10.7 \%$ \\
& $31-40$ & 65 & $31.7 \%$ \\
Género & $41-50$ & 72 & $35.1 \%$ \\
& $51-65$ & 46 & $22.4 \%$ \\
Nivel de enseña & Femenino & 123 & $60 \%$ \\
& Masculino & 82 & $40 \%$ \\
\multirow{3}{*}{ Grado de instrucción } & Inicial & 37 & $18 \%$ \\
& Primaria & 82 & $40 \%$ \\
& Secundaria & 86 & $42 \%$ \\
Situación laboral & Técnico & 11 & $5.4 \%$ \\
& Bachiller & 92 & $44.9 \%$ \\
& Licenciado & 75 & $36.6 \%$ \\
& Magister & 27 & $13.2 \%$ \\
& Contratado & 99 & $48.3 \%$ \\
& Nombrado & 106 & $51.7 \%$ \\
\hline
\end{tabular}

Nota: $\mathrm{n}=205$.

\section{Instrumentos}

Para la recolección de datos se utilizó La Escala Multidimensional de Satisfacción Laboral Docente (EMSLD) fue diseñada por Barraza y Ortega (2009), para evaluar la satisfacción laboral en los docentes a través de 34 ítems que se agrupan en ocho dimensiones; relaciones interpersonales, desempeño profesional, condiciones laborales, valoración del trabajo desarrollado, equipo directivo, participación, factores organizacionales y ambiente físico. Con opciones de respuesta tipo Likert $(1=$ totalmente insatisfecho a $4=$ totalmente satisfecho).

En el presente estudio se analizó la validez y confiabilidad del instrumento obteniendo coeficientes satisfactorios. La validez de contenido fue estudiada por criterio de jueces y el estadístico V de Aiken (Ventura-León, 2019) obteniendo valores superiores a 0.70 (véase Tabla 2). Así mismo, los índices de consistencia interna medidos a través del coeficiente de alfa de Cronbach presentan una adecuada fiabilidad para la escala de satisfacción laboral $(\alpha=.96)$ y sus dimensiones relaciones interpersonales $(\alpha=.63)$, desempeño profesional $(\alpha=.77)$, condiciones laborales $(\alpha=.85)$, valoración del trabajo desarrollado $(\alpha=.71)$, equipo directivo $(\alpha=.85)$, participación $(\alpha=.72)$, factores organizacionales $(\alpha=.88)$ y ambiente físico $(\alpha=.84)$.

El síndrome de burnout fue evaluado a través del inventario de burnout de Maslach (MBI) (Maslach y Jackson, 1981). Se utilizó la versión adaptada y validada por Fernández (2002), consta de 22 ítems. Este inventario permite medir el síndrome a través de sus tres dimensiones; agotamiento emocional (9 ítems), despersonalización (5 ítems) y realización personal ( 8 ítems). Con opciones de respuesta tipo Likert $(0=$ nunca, $1=$ alguna vez al año o menos, $2=$ una vez al mes o menos, $3=$ algunas veces al mes, $4=$ una vez por semana, $5=$ algunas veces a la semana, 6=todos los días). En cuanto a su validez, el instrumento fue estudiado por Fernández (2002), a 
través de la validez de contenido por criterio de jueces y el estadístico V de Aiken obteniendo valores superiores a .70 (.89 a 1). El análisis de confiabilidad en este estudio fue a través del coeficiente de alfa de Cronbach obteniendo resultados confiables para agotamiento emocional $(\alpha=.86)$, despersonalización $(\alpha=.63)$ y $(\alpha=.70)$ para realización personal.

\section{Procedimiento}

Para la recolección de datos, se gestionó los permisos con las autoridades respectivas de las instituciones educativas nacionales de la ciudad de Moyobamba. Una vez obtenido el permiso, a través del formulario de google, se enviaron los instrumentos donde se explicó los fines de la investigación y las instrucciones para responder a cada pregunta. El tiempo estimado para responder fue de 15 a 25 minutos. Además, se solicitó la participación voluntaria por medio del consentimiento informado a los participantes, bajo las condiciones de respetar su privacidad y la confidencialidad de la información brindada solo con propósitos académicos e investigación, respetando así los principios éticos.

\section{Análisis de datos}

Para el análisis de los datos se usó el programa estadístico SPSS versión 23 en donde se realizó un análisis descriptivo a fin de obtener las frecuencias de cada variable, además, se realizó la prueba de normalidad mediante la asimetría y curtosis, evidenciando una distribución asimétrica por lo que para el análisis inferencial se decidió utilizar la rho de Spearman.

\section{Resultados}

\section{Análisis descriptivo}

En primer lugar, se realizó el análisis descriptivo para describir los niveles de las variables en los docentes. Los resultados muestran en la Tabla 2, que el 35.1\% de los participantes tienen un nivel alto de satisfacción laboral. Respecto a las dimensiones, se aprecia que la mayoría de docentes se encuentran en un nivel alto en las dimensiones de relaciones interpersonales $(52.7 \%)$ y participación $(50.7 \%)$. En cuanto al factor organizacional el $49.3 \%$ de los docentes se encuentra en un nivel promedio.

Tabla 2.

Niveles de satisfacción laboral y sus dimensiones en los docentes

\begin{tabular}{lcccccc}
\hline \multirow{2}{*}{ Variables } & \multicolumn{2}{c}{ Bajo } & \multicolumn{2}{c}{ Promedio } & \multicolumn{2}{c}{ Alto } \\
\cline { 2 - 7 } & $\mathrm{f}$ & $\%$ & $\mathrm{f}$ & $\%$ & $\mathrm{f}$ & $\%$ \\
\hline Satisfacción laboral & 63 & $30.7 \%$ & 70 & $34.1 \%$ & 72 & $35.1 \%$ \\
Relaciones interpersonales & 15 & $7.3 \%$ & 82 & $40 \%$ & 108 & $52.7 \%$ \\
Desarrollo personal & 58 & $28.3 \%$ & 63 & $30.7 \%$ & 84 & $41 . \%$ \\
Condiciones laborales & 57 & $27.8 \%$ & 71 & $34.6 \%$ & 77 & $37.6 \%$ \\
Valor del trabajo & 23 & $11.2 \%$ & 80 & $39 . \%$ & 102 & $49.8 \%$ \\
Equipo directivo & 59 & $28.8 \%$ & 59 & $28.8 \%$ & 87 & $42.4 \%$ \\
Participación & 5 & $2.4 \%$ & 96 & $46.8 \%$ & 104 & $50.7 \%$ \\
Factor organizacional & 30 & $14.6 \%$ & 101 & $49.3 \%$ & 74 & $36.1 \%$ \\
Ambiente físico & 38 & $18.5 \%$ & 77 & $37.6 \%$ & 90 & $43.9 \%$
\end{tabular}

Además, en la Tabla 3 se observa que el $37.4 \%$ de docentes mujeres presentan alta satisfacción laboral, a diferencia del $40.2 \%$ de docentes varones que muestran una satisfacción laboral promedio. En relación con el nivel enseñanza, el $45.9 \%$ de los docentes que enseñan al 
nivel inicial y el $40.7 \%$ que enseñan al nivel secundario presentan una alta satisfacción laboral, en contraste del $40.2 \%$ de docentes que enseñan al nivel primario que se ubican en un nivel promedio. Por último, el 39.4\% de docentes contratados presentan alta satisfacción laboral, por su parte el $38.7 \%$ de docentes nombrados muestran un nivel promedio.

Tabla 3.

Nivel de satisfacción laboral según datos sociolaborales

\begin{tabular}{llccc}
\hline & & \multicolumn{3}{c}{ Satisfacción laboral } \\
\cline { 3 - 5 } & & Bajo & Promedio & Alto \\
\hline \multirow{3}{*}{ Género } & & $\%$ & $\%$ & $\%$ \\
& Masculino & $28 \%$ & $40.2 \%$ & $31.7 \%$ \\
& Femenino & $32.5 \%$ & $30.1 \%$ & $37.4 \%$ \\
\multirow{3}{*}{ Situación laboral } & $16.2 \%$ & $37.8 \%$ & $45.9 \%$ \\
& Inicial & $35.4 \%$ & $40.2 \%$ & $24.4 \%$ \\
& Primaria & $32.6 \%$ & $26.7 \%$ & $40.7 \%$ \\
& Secundaria & $31.3 \%$ & $29.3 \%$ & $39.4 \%$ \\
& Contratado & $30.2 \%$ & $38.7 \%$ & $31.1 \%$ \\
\hline
\end{tabular}

En relación con la Tabla 4, se aprecia que el $37.6 \%$ de los docentes presentan un alto nivel de agotamiento emocional y un $30.2 \%$ presentan un nivel moderado. Respecto a la dimensión despersonalización, se aprecia que el $48.3 \%$ de los encuestados se encuentran en el nivel bajo mientras que el $39.5 \%$ en el nivel alto. Además, se observa que el $36.1 \%$ de los participantes tienen niveles bajos de realización personal y un $27.3 \%$ se ubica en el nivel promedio.

Tabla 4.

Niveles de los componentes del síndrome de burnout en los docentes

\begin{tabular}{lcccccc}
\hline \multirow{2}{*}{ Variables } & \multicolumn{2}{c}{ Bajo } & \multicolumn{2}{c}{ Promedio } & \multicolumn{2}{c}{ Alto } \\
\cline { 2 - 7 } & $\mathrm{f}$ & $\%$ & $\mathrm{f}$ & $\%$ & $\mathrm{f}$ & $\%$ \\
\hline Agotamiento emocional & 66 & $32.2 \%$ & 62 & $30.2 \%$ & 77 & $37.6 \%$ \\
Despersonalización & 99 & $48.3 \%$ & 25 & $12.2 \%$ & 81 & $39.5 \%$ \\
Realización personal & 74 & $36.1 \%$ & 56 & $27.3 \%$ & 75 & $36.6 \%$ \\
\hline
\end{tabular}

En la Tabla 5, se aprecia que el $45.5 \%$ de docentes mujeres tienen un alto agotamiento emocional, sin embargo, el $41.5 \%$ presentan altos niveles de realización personal. Por el contrario, el $39 \%$ y el $46.3 \%$ de docentes varones tienen un bajo agotamiento emocional y realización personal respectivamente. Con respecto a la dimensión de despersonalización tanto el $46.3 \%$ de varones y el $49.6 \%$ de mujeres docentes se ubican en un nivel bajo.

Respecto al nivel de enseñanza el 50\% de docentes que enseñan al nivel secundario tienen una baja realización personal. Además, el 54.1\% de docentes que enseñan al nivel inicial, el $45.1 \%$ que enseñan al nivel primario y el $48.8 \%$ que enseñan al nivel secundario tienen bajos niveles de despersonalización.

Finalmente, el $47.5 \%$ de los docentes contratados presentan un nivel alto de despersonalización a diferencia del $53.8 \%$ de docentes nombrados que presentan un nivel bajo. Además, el $38.4 \%$ de docentes contratados presentan baja realización personal a diferencia del $37.7 \%$ de docentes nombrados que tienen alta realización personal. 
Tabla 5.

Niveles de los componentes del síndrome de burnout y datos sociolaborales en docentes

\begin{tabular}{lcccccccccc}
\hline & \multicolumn{3}{c}{ Agotamiento emocional } & \multicolumn{3}{c}{ Despersonalización } & \multicolumn{3}{c}{ Realización personal } \\
\cline { 2 - 10 } & $\begin{array}{c}\text { Bajo } \\
\%\end{array}$ & $\begin{array}{c}\text { Promedio } \\
\%\end{array}$ & $\begin{array}{c}\text { Alto } \\
\%\end{array}$ & $\begin{array}{c}\text { Bajo } \\
\%\end{array}$ & $\begin{array}{c}\text { Promedio } \\
\%\end{array}$ & $\begin{array}{c}\text { Alto } \\
\%\end{array}$ & $\begin{array}{c}\text { Bajo } \\
\%\end{array}$ & $\begin{array}{c}\text { Promedio } \\
\%\end{array}$ & $\begin{array}{c}\text { Alto } \\
\%\end{array}$ \\
\hline $\begin{array}{l}\text { Género } \\
\text { Masculino }\end{array}$ & 39 & 35.4 & 25.6 & 46.3 & 15.9 & 37.8 & 46.3 & 25.6 & 28 \\
$\begin{array}{l}\text { Femenino } \\
\text { Nivel }\end{array}$ & 27.6 & 26.8 & 45.5 & 49.6 & 9.8 & 40.7 & 30.1 & 28.5 & 41.5 \\
enseñanza & & & & & & & & & \\
Inicial & 37.8 & 18.9 & 43.2 & 54.1 & 2.7 & 43.2 & 29.7 & 21.6 & 48.6 \\
Primaria & 30.5 & 32.9 & 36.6 & 45.1 & 13.4 & 41.5 & 25.6 & 31.7 & 42.7 \\
Secundaria & 31.4 & 32.6 & 36 & 48.8 & 15.1 & 36 & 50 & 25.6 & 24.4 \\
$\begin{array}{l}\text { Situación } \\
\text { laboral }\end{array}$ & & & & & & & & & \\
Contratado & 29.3 & 29.3 & 41.4 & 42.4 & 10.1 & 47.5 & 38.4 & 27.3 & 34.3 \\
Nombrado & 34.9 & 31.1 & 34 & 53.8 & 14.2 & 32.1 & 34.9 & 27.4 & 37.7 \\
\hline
\end{tabular}

Nota. $\mathrm{X}^{2} / \mathrm{gl}=$ medida de parsimonia; RMSEA= raíz del error cuadrático media de aproximación; $\mathrm{CFI}=$ índice de ajuste comparativo; TLI= índice Tucker Lewis; $S R M R=$ raíz promedio de residuos estandarizados.

\section{Prueba de normalidad}

Respecto a los análisis descriptivos de las escalas administradas, se obtuvo la media (M), la desviación estándar (DE), la asimetría y la curtosis (Tabla 6). Los valores de asimetría y curtosis indican que siguen una distribución asimétrica, dado que se encuentran por encima del rango +/1.5 (Pérez y Medrano, 2010). Estos indicadores permitieron el uso de la rho de Spearman para la contratación de hipótesis.

Tabla 6.

Análisis descriptivo de la escala multidimensional de satisfacción laboral y síndrome de burnout

\begin{tabular}{lllll}
\hline Variables & Media & D.E. & Asimetría & Curtosis \\
\hline Satisfacción laboral & 103.3 & 14.4 & -.912 & 1.56 \\
Agotamiento emocional & 17.5 & 10.9 & .464 & -.542 \\
Despersonalización & 4.49 & 5.46 & 1.41 & 1.55 \\
Realización personal & 5.59 & 5.72 & .996 & .165 \\
\hline
\end{tabular}

\section{Análisis de correlación}

En la Tabla 7 se aprecia una relación significativa inversa y moderada entre satisfacción laboral $\mathrm{y}$ agotamiento emocional $(\mathrm{rho}=-.315, \mathrm{p}=.000)$, es decir los docentes que presentan un elevado cansancio emocional, muestran niveles bajos niveles de interés y motivación laboral. Por otro lado, se aprecia que existe una relación significativa inversa y débil entre despersonalización y satisfacción laboral ( $\mathrm{r}=-.252, \mathrm{p}=.000)$, es decir los docentes que presentan pérdida de sensibilidad y empatía expresaran una evaluación negativa hacia su trabajo. Finalmente se aprecia que existe una relación significativa inversa y débil entre el grado de realización personal y el nivel de satisfacción laboral $(\mathrm{r}=-.254, \mathrm{p}=.000)$, es decir los docentes que presentan niveles alto de deterioro de sus capacidades y éxito en la realización de su trabajo, a su vez presentaran una actitud negativa hacia su labor. 
Tabla 7.

Correlaciones entre satisfacción laboral y componentes de síndrome de burnout

\begin{tabular}{lll}
\hline & \multicolumn{2}{c}{ Satisfacción laboral } \\
\cline { 2 - 3 } Síndrome de Burnout & rho & $\mathrm{p}$ \\
\hline Agotamiento emocional & $-.315^{* *}$ & 0.00 \\
Despersonalización & $-.252^{* *}$ & 0.00 \\
Realización personal & $-.254^{* *}$ & 0.00 \\
\hline
\end{tabular}

\section{Discusión}

La presente investigación tuvo como objetivo determinar la relación que existe entre la satisfacción laboral y los componentes del síndrome de burnout en los docentes que laboran en instituciones educativas nacionales de la ciudad de Moyobamba durante el confinamiento por la pandemia COVID-19.

De acuerdo a los resultados, se encontró que existe una relación significativa inversa y moderada entre satisfacción laboral y agotamiento emocional, es decir los docentes que presentan bajos niveles de interés y motivación laboral, muestran también un elevado cansancio emocional. Estos hallazgos coinciden con los resultados encontrados tanto en docentes de educación básica en Noruega (Skaalvik y Skaalvik, 2020), Turquía (Atmaca et al., 2020) y Chile (Jiménez et al., 2012), como también en docentes de educación superior (Kamneva, et al., 2019).

Asimismo, Malander (2016) y Menghi y Oros (2014) coinciden que el cansancio emocional, la debilidad y la fatiga son predispuestos por una insatisfacción laboral, tanto extrínseca como intrínseca en los docentes, siendo los factores extrínsecos como la disconformidad con el salario, una jornada laboral extensa, escasas pausas para descansar y trabajo poco flexible, los que predicen niveles altos de agotamiento emocional y los factores intrínsecos como la motivación y autorrealización los que reducen tales síntomas en los docentes. Estos resultados son respaldados desde la teoría organizacional (Cox et al., 1993 y Winnubst, 1993). Así mismo, Aldrete-Rodríguez et al. (2011) encontraron que el agotamiento emocional fue una de las dimensiones que más se relaciona con los diferentes elementos de satisfacción laboral. En las mujeres se asoció con insatisfacción con la supervisión, ambiente de trabajo, prestaciones y aspectos intrínsecos de la tarea. En los hombres se relaciona con supervisión, prestaciones y participación. Por su parte, Smetackova et al. (2019) encontraron que los docentes que tuvieron niveles bajos de agotamiento emocional presentaron no sólo niveles altos de satisfacción laboral, sino también mayor autoeficacia y uso frecuente de estrategias de afrontamiento positivas, evidenciando estos últimos como factores protectores contra el agotamiento. Además, añaden que el apoyo social promueve la satisfacción laboral y previene el síndrome de burnout, en especial, según Soto-Rosales y González-Losada, (2018), reduce los síntomas del agotamiento emocional.

Por otro lado, se aprecia que existe una relación significativa inversa y débil entre despersonalización y satisfacción laboral, es decir los docentes que presentan pérdida de sensibilidad y empatía expresarán una evaluación negativa hacia su trabajo, de la misma forma, Skaalvik y Skaalvik (2020), Hermosa (2006), Malander (2016) y Jiménez et al. (2012) coinciden con tales resultados. Frente a esto, Arias, Sánchez y Ceballos (2017) encontraron que los docentes que sienten que su trabajo les satisface personalmente, ya sea porque les permite desarrollarse y alcanzar sus metas o porque disfrutan de su trabajo tienen menos probabilidad de desarrollar síntomas de despersonalización. Además, Aldrete-Rodríguez et al. (2011) hallaron que la dimensión de despersonalización se relaciona negativamente con diferentes aspectos de satisfacción laboral como la supervisión, el ambiente de trabajo, la insatisfacción con las prestaciones y con la participación. 
Respecto a la relación entre realización personal y satisfacción laboral, los datos recogidos y analizados permiten concluir que existe una relación significativa inversa y débil $(\mathrm{rho}=-.254, \mathrm{p}=.0$ ), es decir los docentes que presentan niveles altos de deterioro de sus capacidades y sentimiento de fracaso en la realización de su trabajo, a su vez presentaran una actitud negativa hacia su labor. De manera similar, Malander (2016) y Menghi y Oros (2014) encontraron que los docentes con insatisfacción laboral presentan una baja realización personal, sobre todo aquellos docentes que presentan insatisfacción en aspectos intrínsecos como posibilidad de logro, reconocimiento de los superiores y colegas por el desempeño, motivación y autonomía. Por lo contrario, Jiménez et al. (2012) refieren que los docentes que muestren competencia y éxito en su labor también presentarán satisfacción laboral en aspectos como la supervisión, las prestaciones, la participación y el ambiente físico.

Se concluye que, los docentes que presentan elevado cansancio emocional, pérdida de sensibilidad y empatía, deterioro de sus capacidades y sentimiento de fracaso muestran a su vez insatisfacción laboral. Por lo tanto, es necesario seguir realizando investigaciones en el campo de la psicología organizacional.

Una de las limitaciones del presente estudio, es que su naturaleza es correlacional, por lo que se recomienda, que futuras investigaciones analicen una relación de causalidad entre las variables, además de tener un mayor número de participantes. Asimismo, realizar trabajos experimentales y pre-experimentales, puesto que existe la necesidad de implementar programas de intervención con el fin de reducir el burnout que presentan los docentes.

\section{Referencias}

Aldrete-Rodríguez, M., Aranda-Beltrán, C., Valencia-Abundiz, S., \& Salazar-Estrada, J. (2011). Satisfacción laboral y síndrome burnout en docentes de secundaria. Revista de Educación y Desarrollo, http://www.cucs.udg.mx/revistas/edu_desarrollo/anteriores/17/017_Aldrete.pdf

Arias, W., Huamani, J., \& Ceballos, K. (2019). Síndrome de Burnout en profesores de escuela y universidad: un análisis psicométrico y comparativo en la ciudad de Arequipa. Propósitos y Representaciones, 7(3), 72. https://doi.org/10.20511/pyr2019.v7n3.390

Arias, W., Sánchez, M., \& Ceballos, K. (2017). Síndrome De Burnout, Satisfacción Laboral Y Relaciones interpersonales en profesores de una institución educativa de Arequipa. Acta Psicológica Peruana, 2(1), 118-139. http://revistas.autonoma.edu.pe/index.php/ACPP/article/view/72

Atmaca, C., Rizaoglu, F., Türkdogan, T. y Yaylı, D. (2020). An emotion focused approach in predicting teacher burnout and job satisfaction. Teaching and Teacher Education, 90, 103025. https://doi.org/10.1016/j.tate.2020.103025

Barraza, A., \& Ortega, F. (2009). Satisfacción Laboral en Instituciones Formadoras Docentes. Diálogos Educativos, 17(January), 4-17. https://dialnet.unirioja.es/descarga/articulo/3039065.pdf

Cantón, I., \& Téllez, S. (2016). La satisfacción laboral y profesional de los profesores. Revista Lasallista de Investigación, 13(1), 214-226. https://www.redalyc.org/pdf/695/69545978019.pdf

Cavalcante J. (2004). Satisfacción en el trabajo de los directores de escuelas secundarias públicas de la región de Jacobina (Tesis Doctoral). https://www.tdx.cat/bitstream/handle/10803/5042/jjcs1de1.pdf

Cox, T., Kuk, G. y Leiter, M. (1993). Burnout, health, work stress, and organizational healthiness. En W. B. Schaufeli, C. Maslach y T. Marek (Eds.), Professional burnout: Recent developments in theory and research (pp. 177-193). London: Taylor \& Francis

Díaz, C., \& Barra, E. (2017). Resiliencia y satisfacción laboral en profesores de colegios municipales y particulares subvencionados de la comuna de Machalí. Estudios Pedagógicos, 1, 75-86. https://scielo.conicyt.cl/pdf/estped/v43n1/art05.pdf 
Duche, A. B., Gutiérrez, O. A., \& Paredes, F. M. (2019). Satisfacción laboral y compromiso institucional en docentes universitarios peruanos. Conrado, 15(70), 15-24. http://scielo.sld.cu/scielo.php?script=sci_arttext\&pid=S1990$86442019000500015 \& \operatorname{lng}=$ es\&tlng=es.

Fernández, M. (2002). Desgaste psíquico (burnout) en profesores de educación primaria de Lima metropolitana. Persona: Revista de La Facultad de Psicología, 5, 27-66. https://dialnet.unirioja.es/servlet/articulo?codigo $=2881042$

Fondo de las Naciones Unidas para la Infancia. (2020). Educación en pausa: Una generación de niños y niñas en América Latina y el Caribe está perdiendo la escolarización debido al COVID-19. https://www.unicef.org/peru/media/9111/file/Educación en pausa.pdf

García, A., Escorcia, C., \& Perez, B. (2017). Síndrome de Burnout y sentimiento de autoeficacia en profesores universitarios. Propósitos y Representaciones, 5(2), 65-126. http://doi.org/10.20511/pyr2017.v5n2.170

Gil-Monte, P. y Peiró, J. (1999). Perspectivas teóricas y modelos interpretativos para el estudio del síndrome de quemarse por el trabajo. Anales de psicología, 15(2), 261-26. https://revistas.um.es/analesps/article/view/30161/29361

Gómez-Perdomo, G. E., Meneses-Higuita, A. C. y Palacio-Montes, M. C. (2017). La satisfacción laboral y el capital psicológico: factores que influyen en el síndrome de burnout. Ansiedad y Estrés, 23, 71-75. http://doi.org/10.1016/j.anyes.2017.09.002

González-Losada, S. (2018). Las Estrategias Didácticas En La Práctica Docente Universitaria. Profesorado, Revista de Currículum y Formación Del Profesorado, 22(2). https://doi.org/10.30827/profesorado.v22i2.7728

Hermosa, A. (2006). Satisfacción laboral y síndrome de "Burnout" en profesores de educación primaria y secundaria. Revista Colombiana de Psicología, 15, 81-89. https://www.redalyc.org/pdf/804/80401509.pdf

Jiménez, A., Gutiérrez, M., \& Miranda, E. (2012). Burnout, apoyo social y satisfacción laboral en docentes. Psicología Escolar e Educacional, 16(1), 125-134. https://doi.org/10.1590/S1413-85572012000100013

Kamneva, E., Polevaya, M., Popova, A., Simonova, M., Butyrin, G. (2019). Job satisfaction and professional burnout of high school teachers. International Journal of Applied Exercise Physiology, 1(2.1), 751-755. https://www.elibrary.ru/item.asp?id=38542717

Malander, N. (2016). Síndrome de Burnout y Satisfacción Laboral en Docentes de Nivel Secundario. Ciencia \& Trabajo, 18(57), 177-182. https://doi.org/10.4067/S071824492016000300177

Maslach, C., \& Jackson, S. E. (1981). The measurement of experienced burnout. Journal of Organizational Behavior, 2(2), 99-113. https://doi.org/10.1002/job.4030020205

Mejia, C., Chacon, J., Enamorado-Leiva, O., Garnica, L., Chacón-Pedraza, S. Garcia-Espinoza, Y. (2019). Factores asociados al estrés laboral en trabajadores de seis países de Latinoamérica. Revista de la Asociación Española de Especialistas en Medicina del Trabajo, 28(3), 204-2011. https://scielo.isciii.es/pdf/medtra/v28n3/1132-6255-medtra28-03-204.pdf

Menghi, M., \& Oros, L. (2014). Satisfacción laboral y Síndrome de Burnout en docentes de nivel primario. Revista de Psicología, 10(20), https://core.ac.uk/download/pdf/80371609.pdf

MINEDU. (2020). Resolución Ministerial 088-2020-MINEDU. In Gobierno del Perú (p. 27). https://cdn.www.gob.pe/uploads/document/file/574993/RVM_N_088-2020MINEDU.pdf

Pérez, E., \& Medrano, L. (2010). Análisis factorial exploratorio: Bases conceptuales y metodológicas. Revista Argentina de Ciencias Del Comportamiento, 2(1889), 58-66. https://dialnet.unirioja.es/descarga/articulo/3161108.pdf

Robalino, M., \& Körner, A. (2005). Condiciones de trabajo y salud docente: estudios de casos en Argentina, Chile, Ecuador, México, Perú y Uruguay (Issue October 2015). https://unesdoc.unesco.org/ark:/48223/pf0000142551 
Skaalvik, E. y Skaalvik, S. (2020) Teacher burnout: relations between dimensions of burnout, perceived school context, job satisfaction and motivation for teaching. A longitudinal study. Teachers and Teaching, 26(7-8), 602-616. https://doi.org/10.1080/13540602.2021.1913404

Smetackova, I., Viktorova, I., Pavlas Martanova, V., Pachova, A., Francova, V., \& Stech, S. (2019). Teachers between job satisfaction and burnout syndrome: What makes difference in Czech elementary schools. Frontiers in Psychology, 10(OCT), 1-8. https://doi.org/10.3389/fpsyg.2019.02287

Soto-Rosales, A. y González-Losada, S. (2018). Satisfacción laboral y desgaste profesional en trabajadores de servicios sociales de atención a la infancia. Epistemes, 8 (14), 80-107. https://doi.org/10.30827/tsg-gsw.v8i14.7222

Tomé, D. y Ceinos, C. (2017). Aproximación a la influencia de la satisfacción laboral y el conflicto de rol en el síndrome del burnout en docentes de secundaria: un estudio de caso. Innovación Educativa, 27, https://revistas.usc.gal/index.php/ie/article/view/3989/4743

Ventura-León, J. (2019). De regreso a la validez basada en el contenido. Adicciones, 20(March), 2-5. https://doi.org/10.20882/adicciones.1213

Winnubst, J. A. (1993). Organizational structure, social support, and burnout. En W. B. Schaufeli, C. Maslach y T. Marek (Eds.), Professional burnout: Recent developments in theory and research (pp. 151-162). London: Taylor \& Francis 